\title{
OP39
}

\section{EXPLORING THE PLEASANT SIDE OF GLARE IN THE LED ERA}

\author{
Dragan Sekulovski et al.
}

DOI 10.25039/x46.2019.OP39

from

CIE x046:2019

Proceedings

of the

29th CIE SESSION

Washington D.C., USA, June 14 - 22, 2019

(DOI 10.25039/x46.2019)

The paper has been presented at the 29th CIE Session, Washington D.C., USA, June 14-22, 2019. It has not been peer-reviewed by CIE.

(C) CIE 2019

All rights reserved. Unless otherwise specified, no part of this publication may be reproduced or utilized in any form or by any means, electronic or mechanical, including photocopying and microfilm, without permission in writing from CIE Central Bureau at the address below. Any mention of organizations or products does not imply endorsement by the CIE.

This paper is made available open access for individual use. However, in all other cases all rights are reserved unless explicit permission is sought from and given by the CIE.

CIE Central Bureau

Babenbergerstrasse 9

A-1010 Vienna

Austria

Tel.: +4317143187

e-mail: ciecb@cie.co.at

www.cie.co.at 


\title{
EXPLORING THE PLEASANT SIDE OF GLARE IN THE LED ERA
}

\author{
Sekulovski, D. ${ }^{1}$, Perz, M. ${ }^{1}$, Vissenberg, M.C.J.M. ${ }^{1}$ \\ ${ }^{1}$ Signify Research, Eindhoven, NETHERLANDS \\ Dragan.sekulovski@signify.com
}

DOI $10.25039 / \times 46.2019 .0 P 39$

\begin{abstract}
An experiment is reported showing the dependency of sparkle and glare perception on luminance and area of a light source. The experimental conditions are chosen such that the results can be applied to design aesthetically pleasant LED sources. Similar to results of previous studies, probabilities of sparkle and glare perception are conveniently expressed in the luminance-area parameter space.
\end{abstract}

Keywords: Visual perception, Sparkle, Glare, LED sources

\section{Introduction}

Surfaces with a high luminance, i.e. luminance much higher than the one the observer is adapted to, generally produce unwanted visual effects, grouped under the term glare (CIE, 1995). From the loss of task performance due to disability glare, the irritation from discomfort glare, to the mysterious effects of overhead glare, we seldom speak of glare as pleasant. Our view on the matter was dramatically changed through a series of papers by Akashi starting around the year 2000 (Akashi et al., 2000). He introduced "Sparkle, the good glare" as a concept that naive observers seem to understand without training (Akashi et al., 2006). A definition used in the experiments was "Sparkle is defined as smaller luminous elements often found to be aesthetically pleasing". Examples used included chandelier glass elements, Christmas lights, and reflections from shimmering water.

In the experiments reported by Akashi, an array of circular openings with a varying area in an opaque material was positioned in front of a light source with a tuneable intensity. Observers could then classify the resulting brightness impression in one of four different categories labelled by the terms "dull", "bright", "sparkling", and "glaring". A very useful way of fitting and using the results of the experiments is as a probability of observing sparkle or glare for different points in the log(solid angle)/ log(luminance) parameter space.

As revolutionary as those experiments are, there is one problem limiting their applicability to LED based light sources and that is the range of luminances used in the experiment. As the fluorescent tubes were used to generate the light stimuli, the highest luminance was less than $10000 \mathrm{~cd} / \mathrm{m}^{2}$, far from the levels that we regularly see in LED based light sources. To enrich the data on the perception of both pleasant and unpleasant glare we carried out an experiment looking at luminances up to $3^{*} 10^{6} \mathrm{~cd} / \mathrm{m}^{2}$. The reported results can be used to help design LED sources that give raise to the perception of sparkle and thus are more pleasant to look at.

\section{Experimental}

The experimental procedure was similar to the one used by Akashi, with the most notable difference being the hardware used. Furthermore, in contrast with the prior studies, instead of classification of the impression of the stimulus, we asked the participants to directly judge if the stimulus was perceived as "glary" and "sparkly" in two different sessions, implying that the two effects are not mutually exclusive.

\subsection{Set up and stimuli}

An array of $64(8 \times 8)$ so called "chip on board $(\mathrm{CoB})$ " modules were connected to a computer controllable power supply (Delta SM3300). The modules had a color temperature of $2700 \mathrm{~K}$, $\mathrm{CRI}$ of 90 , and each produced up to 2000 lumen, giving a possible maximum output above 
60000 lumen and luminance of the chips of up to $3^{*} 10^{6} \mathrm{~cd} / \mathrm{m}^{2}$. The power supply was controlled using custom software that changed the driving current of the light source modules. The modules were mounted on an over dimensioned cooling plate $(60 \mathrm{~cm} \times 60 \mathrm{~cm})$ and good thermal contact was insured. The LED modules used in the experiment are shown in Figure 1(a).

To create light stimuli of varied luminous areas, a set of differently sized circular openings were laser-drilled in aluminium plates (4 columns and 8 rows), aligned to the centers of the LED modules. The openings had diameters of $1.3,1.6,2.4,3.2,4.8$, and $6.4 \mathrm{~mm}$ with a horizontal pitch of $70 \mathrm{~mm}$, and vertical pitch of $76 \mathrm{~mm}$ between the centers of the openings. Viewed from the distance of $3.05 \mathrm{~m}$ these corresponded to $-6.85-6.67-6.31-6.06-5.71-5.46$ log steradians. The metal plates were manually positioned in front of the light modules in a slot that assured good alignment. Ten different luminances were used in the experiment, being 1445, 3372, 7462, $10^{4}, 3.2 * 10^{4}, 10^{5}, 3.2 * 10^{5}, 10^{6}, 2 * 10^{6}$, and $3 * 10^{6} \mathrm{~cd} / \mathrm{m}^{2}$.

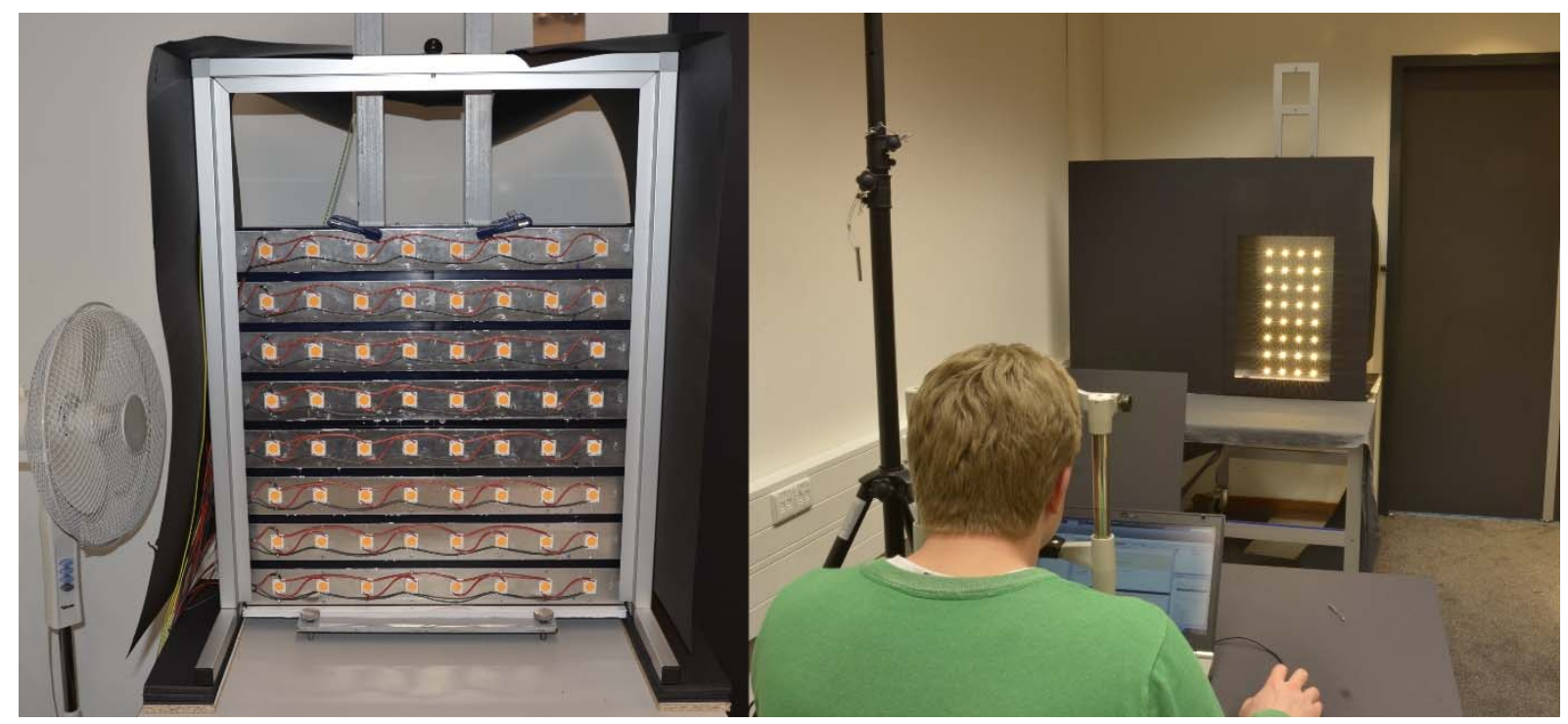

Figure 1 - Experimental set-up (a) a matrix of 64 dimmable LEDs, mounted on a heat sink, (b) a participant during the experiment, looking at a light stimulus, where aluminium plate with drilled openings ( 4 columns $\times 8$ rows) is positioned in front of the LED modules.

\subsection{Participants}

20 participants took part in the experiment, 10 males and 10 females with an age range of 25 to 65. All participants were recruited using an external agency and had no prior professional experience with lighting and lighting design and were west Europeans.

\subsection{Procedure}

The participants were seated on a fixed position $3.05 \mathrm{~m}$ from the light stimulus using a chin rest in a room with additional office lighting with the same color temperature as the stimulus. The illuminance at the eye of the participants with the fixed adaptation stimulus was $16 \mathrm{Ix}$.

The participants were introduced to the experiment and signed the informed consent form. They were given verbal and written definitions of sparkle and glare, and these were similar to the definitions used by Akashi: "Sparkle is defined as smaller luminous elements often found to be aesthetically pleasant. Examples: chandelier prisms, Christmas lights, reflections from shimmering water", "Glare is defined as obtrusive unpleasant light directed in your eyes. Examples: reflections of windshields, reflections from calm water when you use your hand to block a portion of the light". Then, the participant had a training session which included stimuli that were clearly glary and clearly sparkly.

During the experiment, all participants saw all stimuli twice. Due to the manual change of the plates, all luminances for a fixed area (i.e. the same aperture diameter) were presented twice in a random order. Then the plate was changed, changing the area. The areas were presented in a random order over the participants. 
To minimize the effect of local and global adaptation, the stimuli were shown for a limited time and a fixed stimulus was shown in between. The light of the stimulus was switched on for two seconds after which the participant was asked to rate the stimulus as sparkly or not by pressing the corresponding keys on a numeric keyboard connected to a laptop. In a separate session, balanced in the order with the sparkle session, the participant was asked to rate the stimulus as glary or not glary using the same procedure. After the answer of the participant, the stimulus was switched to a fixed luminance of $5000 \mathrm{~cd} / \mathrm{m}^{2}$ for 10 seconds, after which the procedure was continued with the next stimulus.

Through the experiment each of the participants saw 120 conditions: 10 (luminance level) $\times 6$ (luminance area) $\times 2$ (repetitions); and it took about 30 min to complete it.

\section{Results}

The data was aggregated into a probability of rating each stimulus given with the (luminance, area) pair as glary or sparkly. The resulting mean detection probabilities are shown in Figure 2 (sparkle) \& Figure 3 (glare), depicted with green dots. These probabilities were further used to fit two generalized linear models(GLMs, e.g. Collett, 2002). The subsequent terms were included in each of the models if they significantly decreased the error $(p<0.001)$, determined by the chi-squared likelihood ratio test. The GLM that best describes the probability of detecting both sparkle and glare in the experiment is defined as:

$$
p \sim L+L^{2}+L^{3}+L^{4}+S+S^{2}+S^{3}+S^{4}+L * S+L^{2} * S+L * S^{2}+L^{3} * S+L * S^{3}+L^{2} * S^{2}
$$

where

$p \quad$ is the probability of detecting sparkle or glare;

$L \quad$ is the luminance of the source;

$S \quad$ is the size of the aperture.

The estimated coefficients of the GLM are given in Table 1.

Table 1 - Estimated coefficients of the GLM model defined in equation (1) that best describe the probability of detecting sparkle and glare measured in the experiment.

\begin{tabular}{c|ccccccccccccccc} 
& intercept & $L$ & $L^{2}$ & $L^{3}$ & $L^{4}$ & $S$ & $S^{2}$ & $S^{3}$ & $S^{4}$ & $L * S$ & $L^{2 * S}$ & $L^{*} S^{2}$ & $L^{3 * S}$ & $L^{*} S^{3}$ & $L^{2 *} S^{2}$ \\
\hline \hline Sparkle & 1.574 & -0.309 & -1.271 & -0.001 & 0.213 & -1.464 & -1.193 & 1.350 & -0.378 & -1.328 & 0.232 & 0.102 & 0.987 & 0.330 & 0.544 \\
Glare & -1.467 & 1.360 & 0.815 & -0.077 & -0.145 & 2.628 & -1.407 & -3.841 & 5.612 & 0.0750 & -0.252 & -0.123 & 1.089 & -0.1936 & -0.393
\end{tabular}

The model used a binomial distribution of the responses and a probit link function. The dependent factors used were the logarithm of the luminance and the logarithm of the solid angle of a single opening as well as their powers and combination of powers up to the fourth degree.

Next to mean measured detection probabilities of sparkle and glare, Figures 2 \& 3 also show the predicted detection probabilities (depicted as solid red lines), resulted from fitting the GLM, defined in equation (1) and using the coefficients shown in Table 1. 

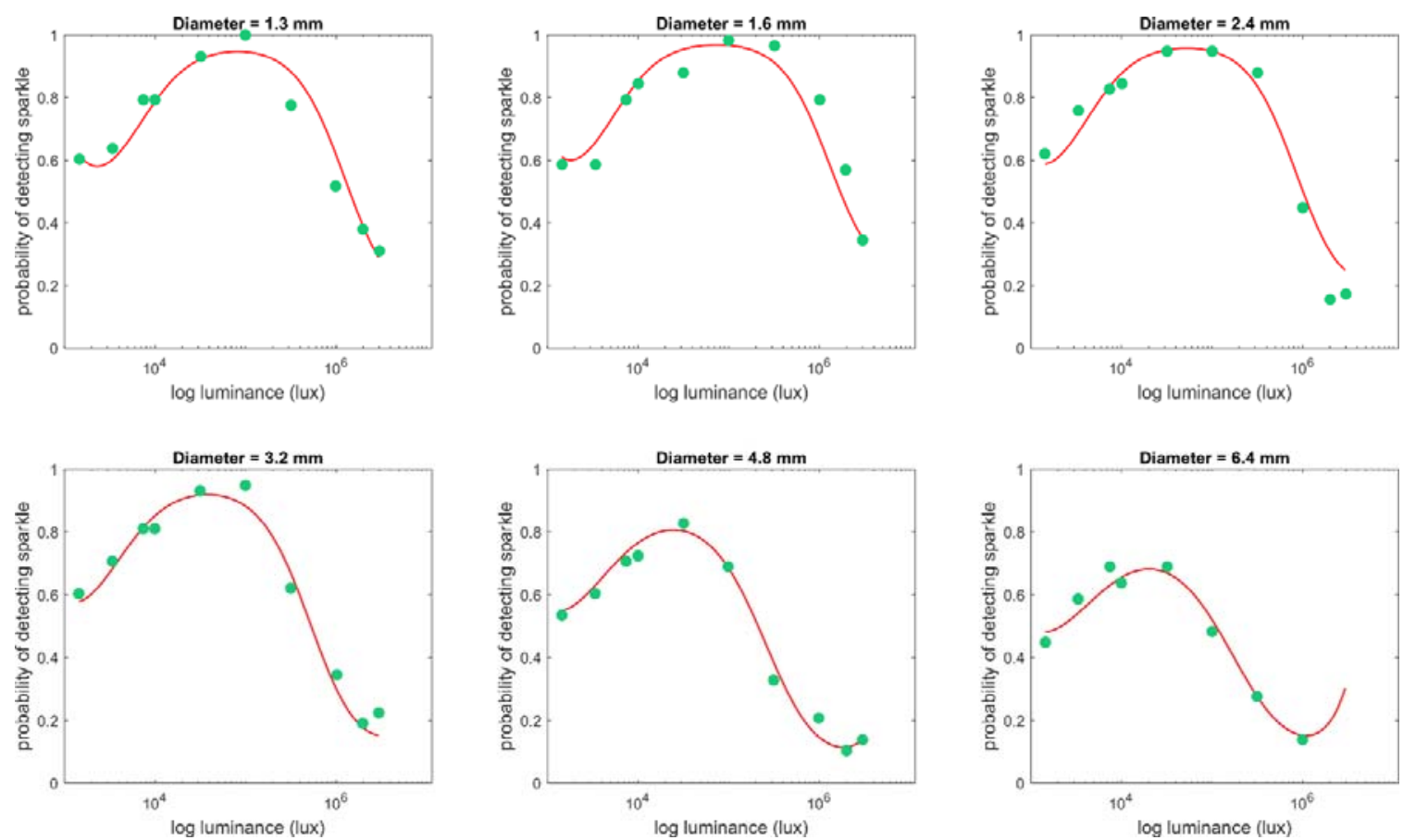

Figure 2 - Depicted as green dots are mean probabilities of sparkle detection (averaged across the participants) as a function of the luminance. The solid red line depicts predicted detection values computed for the generalized linear model, defined in equation (1), using the coefficients values shown in Table 1.
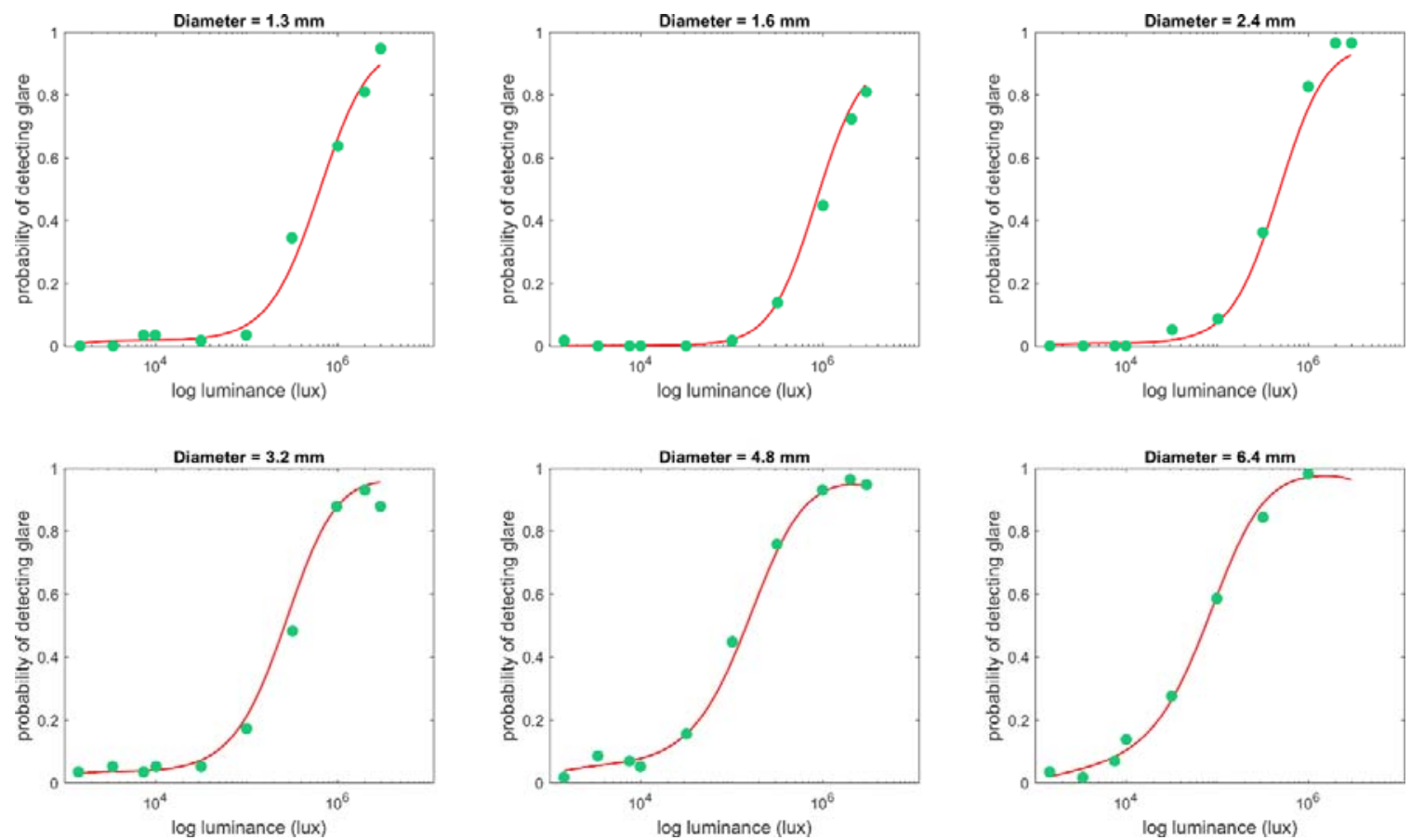

Figure 3 - Depicted as green dots are mean probabilities of glare detection (averaged across the participants) as a function of the luminance. The solid red line depicts predicted detection values computed for the generalized linear model, defined in equation (1), using the coefficients values shown in Table 1.

The results show that the probability of sparkle detection follows an inverted u-shape function of the luminance for all the areas, with the peak around luminance of $10^{5} \mathrm{~cd} / \mathrm{m}^{2}$. Increasing the size of the source generally decreased the probability of sparkle detection. Further, the results 
show an unsurprising tendency that glare probability grows with the increase of luminance and size.

A convenient representation of the results is as contour plot containing the isolines of detection probabilities on the log luminance / log size (solid angle in steradians) plane. Rather than connecting the measured points, the fitted generalized linear model was used, and the resulting diagram is shown in Figure 4 for both sparkle (plotted in green), and glare (in red). Figure 4 shows the interplay between the sparkle and glare perception. Even though these two effects were assumed not to be mutually exclusive, summing the probabilities at any point only slightly exceeds one, implying that there exists a threshold at which the perception changes from sparkle to glare as a function of source area and luminance.

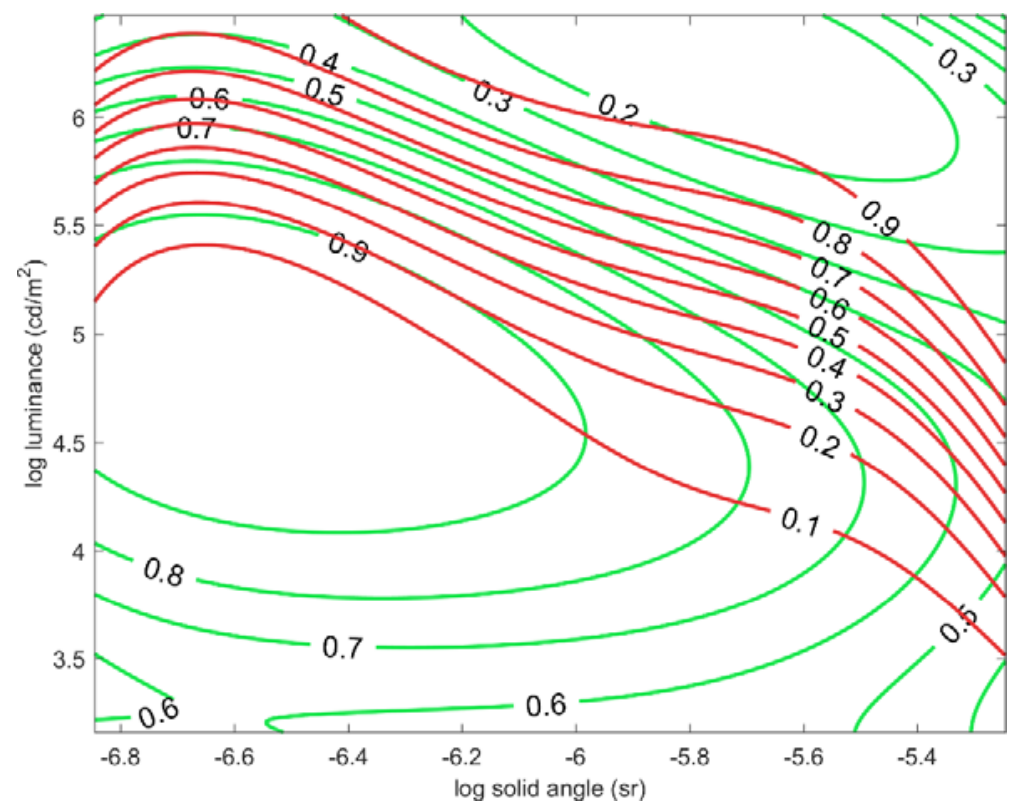

Figure 4 - Iso-probability contour diagram of perceived sparkle (in green) and glare (in red).

It can be read from Figure 4 that the probability of sparkle perception decreases with luminance and area for luminances higher than $3.2 * 10^{5} \mathrm{~cd} / \mathrm{m}^{2}$, but that it increases again for large areas above around $5 \mathrm{~mm}$. It should be stressed that the later is an artefact resulting from fitting the generalized linear model, rather than an actual effect.

\section{Discussion}

The current study was designed and executed to extend the results of Akashi (2006) so that they could be applied to help design aesthetically pleasant LED sources. For easy comparison, Figure 5 shows the previously reported iso-probability diagrams, for low ambient light level of $0.7 \mathrm{Ix}$ and for high level of $160 \mathrm{Ix}$. 

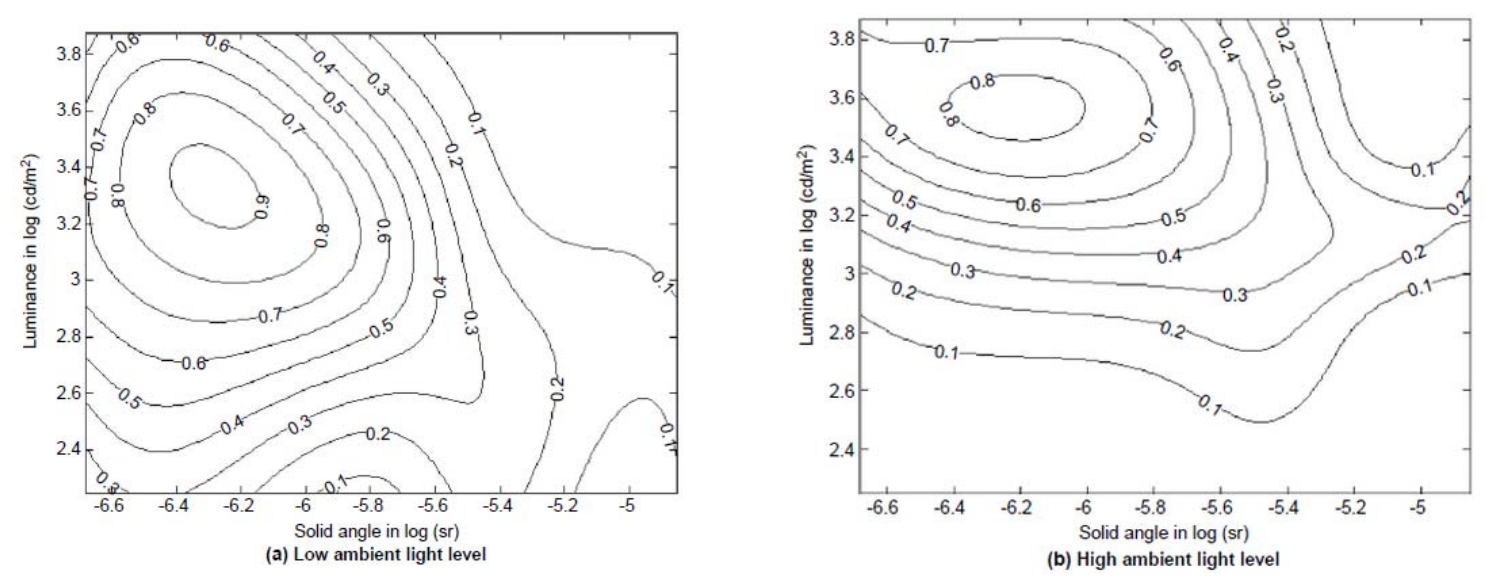

Figure 5 - Iso-probability contour diagrams developed by Akashi (2006) of perceived sparkle, for low and high ambient light levels.

In the current experiment the illuminance measured at subjects eyes' was $16 \mathrm{Ix}$, which is not as dim as Akashi's low ambient condition but also much dimmer than his high ambient condition. We decided to use somewhat intermediate ambient light level, rather than $0.7 \mathrm{Ix}$ or $160 \mathrm{~lx}$ for different reasons. Using $0.7 \mathrm{Ix}$ means that the stimulus provides the only illumination in the environment and we found such conditions unrealistic in general lighting applications. Conversely, adapting to the high level of $160 \mathrm{~lx}$ was found to generally decrease the sparkle perception (i.e. decrease the contrast between the source and the ambient environment) and since we only used one adaptation level we decided to use lower luminance.

Both in Akashi's as well as in our studies the probability of detecting sparkle follows the inverted u-shape function of the luminance and it decreases with the increased areas. However, as it can be seen from the iso-probability contours (Figure 4 \& Figure 5) different optimal luminance areas were found: in our study a peak is positioned at higher luminances of around $10^{5} \mathrm{~cd} / \mathrm{m}^{2}$, and in Akashi's study at $7000 \mathrm{~cd} / \mathrm{m}^{2}$. This significant difference is probably caused by range effects and the adaptation state of the observers.

The probability of glare detection was in general lower for the range of luminances that overlap with the earlier studies, also probably caused by the adaptation state and range effects, stressing the need for control of adaptation in such experiments.

\section{References}

CIE, 1995. Commission Internationale de l'Eclairage. CIE 117:1995. Discomfort Glare in Interior Lighting. Vienna: CIE, 1995

AKASHI, Y. TANABE, Y. AKASHI, I. \& MUKAI, K. 2000. Effect of sparkling luminous elements on the overall brightness impression: A pilot study. International Journal of Lighting Research and Technology, 32(1), 19-26.

AKASHI, Y. MYER, M. A. BOYCE, P. R. 2006. Identifying sparkle. Lighting Research \& Technology, 38 (4), 325-337.

COLLETT, D. 2002. Modeling Binary Data. New York: Chapman \& Hall. 